\title{
QUANTIFICATION OF EFFECTS OF LEVODOPA TREATMENT IN PARKINSONIAN SYNDROMES
}

\author{
GAlli, M.; Cimolin, V.; Vimercati, S.; AlBertini, G.; \\ ONORATI, P. \& DE PANDIS, M. F.
}

Abstract: The purpose of this chapter is to present the experience of the Posture and Motion Laboratory at the "San Raffaele Cassino" in the field of use of Gait Analysis (GA) in patients with Parkinson's disease (PD) and with Progressive Supranuclear Palsy (PSP). In particular, the effects of levodopa medication on PD vs. PSP patients were quantified, comparing the OFF and ON state in the two different pathological conditions, using functional evaluation and GA. Data of the OFF and ON states in 10 PSP patients and 11 PD patients were compared. The results highlighted that the treatment based on Levodopa treatment had significant effects only on PD group (spatio-temporal parameters, kinematics and kinetics); PSP patients revealed no significant changes after the levodopa therapy.

Key words: Parkinson's disease, PSP, gait analysis
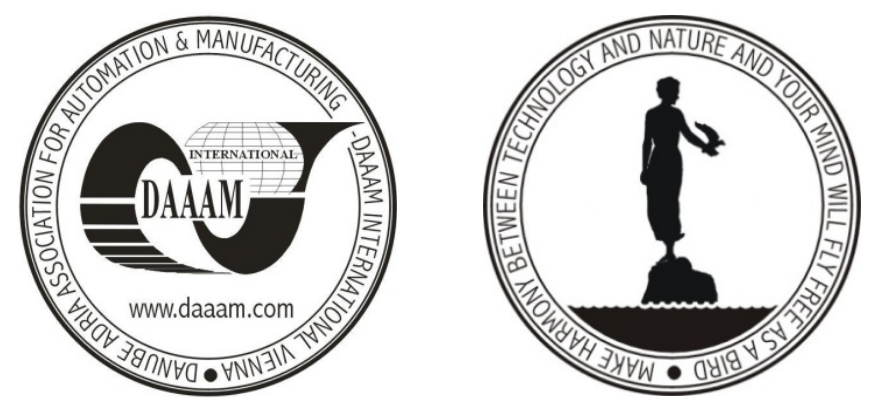

Authors' data: Eng. Galli M[anuela]*, Eng. Cimolin V[eronica]*; Eng. Vimercati S[ara]*; Prof. Albertini G[iorgio]**; Dr. Onorati P[aolo]***; Dr. De Pandis $\mathrm{M}$ [aria] $\mathrm{F}$ [rancesca]***, *Dipartimento di Bioingegneria, Politecnico di Milano, Milano, Italy, ** IRCCS “San Raffaele Pisana” Tosinvest sanita, Roma, Italy; ****San Raffaele Cassino” Institute, Tosinvest Sanita, Cassino, Italy

manuela.galli@polimi.it;veronica.cimolin@polimi.it;giorgio.albertini@sanraffaele.it; paolo.onorati@sanraffaele.it; maria.depandis@sanraffaele.it

This Publication has to be referred as: Galli, M[anuela]; Cimolin, V[eronica]; Vimercati, S[ara]; Albertini, G[iorgio]; Onorati, P[aolo] \& De Pandis, M[aria] F[rancesca] (2010). Quantification of Effects of Levodopa Treatment in Parkinsonian Syndromes, Chapter 06 in DAAAM International Scientific Book 2010, pp. 049-056, B. Katalinic (Ed.), Published by DAAAM International, ISBN 978-3-901509-74-2, ISSN 1726-9687, Vienna, Austria

DOI: 10.2507/daaam.scibook.2010.06 


\section{Introduction}

Three-dimensional motion analysis has turned out to be a powerful tool for a quantitative assessment of the movement. For clinical application, in particular, the breakthrough of three-dimensional motion analysis can be attributed to Gait Analysis (GA), or quantitative analysis of walking, which is generally applied in patients with difficulties in walking. The clinical importance of quantitative analysis of movement is demonstrated by the increasing number of gait laboratories in clinical setting; in particular, one of the most popular clinical applications of gait analysis is the use for diagnostic and rehabilitation planning in treatment of children with Cerebral Palsy (Gage, 2004). With the increasing of the experience and of the clinical needing, Gait Analysis has been used not only in patients with Cerebral Palsy, but also to quantify the gait pattern in other pathological states, both for children and for adults, too. In particular, the experience of the Posture and Motion Laboratory at the "San Raffaele Cassino" (part of the Tosinvest Sanità group) is described in the field of the use of Gait Analysis in patients with Parkinson's Disease (PD) and with Progressive Supranuclear Palsy (PSP).

\section{The use of 3D Gait Analysis in Parkinson's disease and Progressive Supranuclear Palsy}

\subsection{Introduction}

PD and PSP are the most common neurodegenerative disorders that involve the basal ganglia. The neuropathological findings differ, with PD mainly affecting the nigro-striatal pathways, whereas the distribution of degenerative changes in PSP is more widespread in the brain, with the variable involvement of the putamen, pallidum, pyramidal tract, brain stem, cerebellum and spinal cord. Disorders of gait are one of the most common symptoms of PD and PSP. These disorders progressively worsen as the disease advances, severely limiting the patient's quality of life. Making a differential diagnosis is difficult, especially in early disease, and the clinical identification of PSP is important due to the poorer prognosis, different pharmacological considerations and the so far disappointing effects of neurosurgical interventions (Holmberg et al., 2001). In particular, the progression of degenerative symptoms and the response to levodopa therapy are two important clinical features reflecting the diagnosis. Tests using single doses of levodopa or apomorphine are widely used in the diagnostic procedure and for considerations of further treatment in Parkinsonian disorders. The levodopa response is an important part of the clinical diagnostic criteria for PD and PSP and it represents still the golden standard in the treatment of PD. However, the clinical scoring systems used to evaluate the response to levodopa treatment provide only qualitative or semi-quantitative data and in literature detailed quantitative quantification of the effects of levodopa medication in PD and PSP patients is lacking, too.

With regard to characterisation of movement disorders during gait in Parkinsonism, the literature is mainly focused on PD; several studies have highlighted the typical walking pattern of PD patients: shorter stride length, reduced velocity and increased 
stance phase (Morris et al., 2001). Gait analysis has also been used in PD patients to study the effects on locomotion of functional neurosurgery, levodopa therapy, visual cues and rehabilitation. (Morris et al., 2001; Vokaer et al., 2003; Lubik et al., 2006; Moore et al., 2008). However the effects of levodopa medication rarely have been examined systematically though standardised quantitative evaluation, especially in PSP patients, where the evaluation of the effects of levodopa treatment is very poor. So, although clinical rating scales and simple tests of motor function are widely used to assess motor response to therapy, mainly in PD patients, GA, may provide an alternative measure of this response. The aim of this evaluation is to evaluate quantitatively the effects of levodopa medication on PD patients vs. PSP patients, comparing the OFF and ON state in the two different pathological conditions, using 3D-GA.

\subsection{Materials and Methods}

Ten patients with PSP (age: $72.6 \pm 4.8$ years; age-to-onset: $65.1 \pm 5.3$ years) and 11 patients with idiopathic PD (age: $67.6 \pm 6.6$ years; age-to-onset: $58.9 \pm 4.5$ years) referred to the San Raffaele Parkinson's disease Centre, Cassino, Italy, from the beginning of November 2007 through November 2008, matched for age, height and disease severity (Hoehn and Yahr scale and UPDRS part III score at the time of the 3D-GA evaluation), were enrolled in the study. 10 age-matched subjects (age: $66.7 \pm 5.9$ years) with no neurological or orthopaedic conditions that would affect gait were included as controls. The study was approved by the Ethics Research Committee of the San Raffaele Pisana Clinic, Roma, Italy. All subjects gave informed consent to participation in the study. All patients were receiving levodopa therapy at the mean $( \pm \mathrm{SD})$ dosage of $250 \mathrm{mg}( \pm \mathrm{mg})$.

Subjects were excluded if their medical condition was unstable due to neurological, orthopaedic or cardiovascular comorbidity affecting gait. A clinical team made up of a neurologist and a physical therapist examined each patients. Each patient was evaluated in the OFF and in the ON states. The complete evaluation consisted of three components: neurological and functional examination, videotaping and threedimensional GA. Clinical testing was carried out using the Unified Parkinson's Disease Rating Scale (UPDRS) (Fahn et al., 1987). The severity of extrapyramidal symptoms was rated using the motor section of UPDRS (UPDRS III) (Fahn et al., 1987). The functional status was assessed by Functional Independence Measure (FIM) (Keith et a., 1987).

3D-GA was conducted using the following equipments:

- a 9-camera optoelectronic system with passive markers (SMART, BTS, Italy), to measure the kinematic of movement;

- two force platforms (Kistler, $\mathrm{CH}$ ), to obtain the kinetic data of movement (i.e. ground reaction forces);

- 2 TV camera Video system (BTS S.p.A., Milan, Italy) synchronized with the optoelectronic and force platform systems for videorecording.

To evaluate the kinematics of each body segment, markers were positioned as described by Davis (Davis et al., 1991). The subjects, after marker placement, were asked to walk barefoot at their own natural pace (self-selected speed) along a 10- 
metre walkway containing the force platforms at the mid-point. At least five trials were collected for each patient in order to guarantee reproducibility of results. All graphs obtained from GA were normalized as \% of gait cycle. By the analysis of these graphs, some parameters were identified and analysed (like time/distance parameters, angles joint values in specific gait cycle instant, peak values in joint power graphs).

In particular the following parameters were evaluated:

Spatio-temporal parameters:

- duration of the stance phase (as \% of the gait cycle);

- velocity: mean velocity of progression $(\mathrm{m} / \mathrm{s})$;

- Step width: medio-lateral distance between the two foot during double support (mm);

- Anterior step length: longitudinal distance from one foot strike to the next one normalised to subject's height.

\section{Kinematics:}

- the range of motion at pelvic joint on sagittal plane (Prom index) during the gait cycle, expressed in degrees;

- the values of angle of ankle (AIC index), knee (KIC index) and hip joint (HIC index) at the contact of the foot with the ground (i.e. Initial Contact or IC), expressed in degrees;

- the values of maximal ankle dorsiflexion during stance and swing phase (respectively AMSt and AMSw indices), the maximal flexion of the knee (KMSw index) during swing phase, expressed in degrees;

- the values of minimal ankle dorsiflexion in stance phase (AmSt index), knee (KmSt index), hip flexion (HmSt index) during the gait cycle, expressed in degrees;

Kinetics:

Ankle power:

- the maximum value of generated ankle power during terminal stance (maximum value of positive ankle power; APMax index), expressed in $\mathrm{W} / \mathrm{Kg}$. All the previously defined parameters were computed for each subject and then the mean values and standard deviation related to all indices were calculated for the PSP and PD groups and for the healthy group. One-way between groups analysis of variance (ANOVA) was applied for statistical analysis, in order to detect significant differences in the two pathological groups in the OFF state. The assumptions of the ANOVA model were tested by evaluating the fit of the observed data to the normal distribution (Kolmogorov-Smirnov test) and the homogeneity of variances (Levene's test). Specific effects were evaluated by means of the post-hoc comparisons of means the Bonferroni test. Data of the OFF and ON states and of CG were compared with Wilcoxon's tests in the PD and in PSP groups, in order to detect significant changes in the two pathological groups. Statistical significance was set at $\mathrm{p}<0.05$.

\subsection{Results}

All the patients were able to complete both clinical and instrumental evaluation with Gait Analysis. 
Results of neurological and functional evaluations (Table I):

As concerns UPDRS III score, the two pathological groups presented similar UPDRS III scores in OFF state; the effect of the treatment was an improvement of the score in both groups that was significant at statistical level. In terms FIM scores, the two groups revealed different conditions in OFF state and PSP patients were more compromised than PD patients as concerns overall functional disability. In ON condition, both PSP and PD patients increased significantly $(\mathrm{p}<0.05)$ FIM score, evidencing an improvement.

\begin{tabular}{|c|c|c|c|c|}
\hline & \multicolumn{2}{|l|}{ PSP patients } & \multicolumn{2}{|l|}{ PD patients } \\
\hline & OFF state & ON state & OFF state & ON state \\
\hline UPDRS III score & $72.4 \pm 18.4$ & $57.0+15.5+$ & $71.7 \pm 14.4$ & $31.7+9.8+$ \\
\hline FIM & $44.1+19.2$ & $63.7+25.4+$ & $68.0+20.9$ & $99.2+12.9+$ \\
\hline
\end{tabular}

Tab. 1. Clinical characteristics of PSP and PD patients (PSP GROUP and PD GROUP) $(+=\mathrm{p}<0.05$, PSP GROUP versus PD GROUP)

\section{Results of Gait Analysis parameters}

In Tab. II and III the mean values (standard deviation) of all GA parameters considered in this study, spatio-temporal parameters, kinematic and kinetic indices, for pathological groups in the two states (OFF and ON states) and for CG were reported.

Spatio-temporal parameters (Tab. II):

Both groups are characterised by abnormal spatio-temporal parameters in comparison with CG. They both exhibited high duration of stance phase duration, shorter anterior step length, lower velocity of progression and larger step width, if compared to control group. In ON state, the PD subjects displayed significant improvements in terms of stance phase duration, which was in normal range, velocity of progression and anterior step length. On the contrary, no statistical changes occurred in PSP group as concern spatio-temporal indices.

Kinematic parameters (Tab. II):

In terms of kinematic parameters, we can observe that the two pathological groups presented significant differences in the OFF state, with the PSP group more compromised than PD one. Significant differences were evidenced at all lower limb joints (ankle, knee and hip joints): PSP walked with more plantarflexed ankle joint and higher flexion of knee and hip joints during the whole gait cycle, if compared to $\mathrm{PD}$ and control group. The effects of the pharmacological treatment evaluated by the comparison of the OFF vs. ON state in the two pathological groups exhibited that the treatment had significant effects only on PD group. In these patients, a significant improvement of ankle position was displayed in ON state in terms of plantarflexion ability in stance phase (AmSt index), which passed to a normal position. Knee flexextension pattern did not change its position in stance phase, but improved significantly knee flexion in swing phase, reaching values close to normality. As concerns hip position, better extension ability in stance phase was achieved by PD 
Galli, M.; Cimolin, V.; Vimercati, S.; Albertini, G.; Onorati, P. \& De Pandis, M.F.: Quant...

subjects, which exhibited normal hip flex-extension after the treatment. In PSP group no statistically changes appeared in ON state in terms of kinematic parameters, maintaining the values displayed in OFF state.

\begin{tabular}{|c|c|c|c|c|c|}
\hline & \multicolumn{2}{|c|}{ PD GROUP } & \multicolumn{2}{|l|}{ PSP GROUP } & \multirow[t]{2}{*}{ CG } \\
\hline & $\begin{array}{l}\text { OFF } \\
\text { STATE }\end{array}$ & ON STATE & $\begin{array}{l}\text { OFF } \\
\text { STATE }\end{array}$ & ON STATE & \\
\hline \multicolumn{6}{|c|}{ Spatio-temporal parameters } \\
\hline \%stance(\%) & $70.7(8.9)^{* *}$ & $60.5(4.3)^{*}$ & $75.3(9.8)^{* *}$ & $75.9(9.9)^{* *}$ & $59.5(1.4)$ \\
\hline Step length & $0.2(0.2)^{* *}$ & $0.5(0.1)^{*, * *}$ & $0.2(0.1)^{* *}$ & $0.2(0.1)^{* *}$ & $0.9(0.2)$ \\
\hline $\begin{array}{l}\text { Step width } \\
(\mathrm{mm})\end{array}$ & $0.2(0.1)$ & $0.2(0.1)$ & $0.2(0.1)^{* *}$ & $0.2(0.1)^{* *}$ & $0.1(0.1)$ \\
\hline $\begin{array}{l}\text { Velocity } \\
(\mathrm{m} / \mathrm{s})\end{array}$ & $0.4(0.3)^{* *}$ & $0.9(0.3)^{*, * *}$ & $0.2(0.2)^{* *}$ & $0.3(0.3)^{* *}$ & $1.3(0.1)$ \\
\hline \multicolumn{6}{|c|}{ Ankle dorsi-plantarflexion $\left({ }^{\circ}\right)$} \\
\hline AIC & $1.1(5.1)$ & $-1.6(5.6)$ & $-6.0(7.7)^{*++}$ & $-5.5(8.2)^{* *}$ & $1.1(5.7)$ \\
\hline AMSt & 13.5 (4.9) & $15.7(4.1)$ & $8.8(7.6)^{* *+}$ & $7.3(8.9)^{* *}$ & $13.3(5.4)$ \\
\hline $\mathrm{AmSt}$ & $-3.4(6.2)^{* *}$ & $-12.6(7.6)^{*}$ & $-12.9(9.7)^{+}$ & $-12.2(9.7)$ & $-12.0(4.2)$ \\
\hline AMSw & $6.0(2.8)$ & $4.9(5.4)$ & $0.1(9.6)^{* *+}$ & $-0.7(7.3)^{* *}$ & $6.7(4.2)$ \\
\hline \multicolumn{6}{|c|}{ Knee flex-extension $\left({ }^{\circ}\right)$} \\
\hline KIC & $10.1(8.7)^{* *}$ & $9.1(9.2)^{* *}$ & $24.4(9.4)^{* *,+}$ & $26.6(9.3)^{* *}$ & $5.1(4.2)$ \\
\hline $\mathrm{KmSt}$ & $5.2(8.7)$ & $3.8(9.0)$ & $16.8(9.9)^{* *,+}$ & $17.5(9.2)^{* *}$ & $4.3(2.0)$ \\
\hline KMSw & $41.3(8.4)^{* *}$ & $58.9(7.9)^{*}$ & $52.5(9.2)^{* *,+}$ & $49.8(9.0)^{* *}$ & $62.6(6.6)$ \\
\hline \multicolumn{6}{|c|}{ Hip flex-extension $\left(^{\circ}\right)$} \\
\hline HIC & $28.7(9.7)$ & $33.0(7.7)$ & $37.2(6.9)^{* *+}$ & $36.2(6.6)^{* *}$ & $30.3(3.5)$ \\
\hline $\mathrm{HmSt}$ & $4.9(6.8)^{* *}$ & $-5.9(6.8)^{*}$ & $11.8(9.7)^{* *,+}$ & $11.6(9.8)^{* *}$ & $-6.2(4.5)$ \\
\hline \multicolumn{6}{|l|}{ Pelvic tilt $\left({ }^{\circ}\right)$} \\
\hline Prom & $3.4(1.1)$ & $4.1(1.4)$ & $4.1(1.8)$ & $4.5(2.0)$ & $1.6(3.6)$ \\
\hline
\end{tabular}

Tab.2. Comparison of selected spatio-temporal and kinematic parameters (mean and standard deviation) for PD and PSP subjects (PD GROUP and PSP GROUP) and Control Group (CG) $(*=p<0.05$, OFF versus ON states; $+=p<0.05$ OFF state in PD GROUP versus OFF state in PSP GROUP; $* *=p<0.05$ compared with CG)

Kinetic parameters (Tab. III):

As concerns ankle power, in OFF condition the maximum of generated ankle power (APMax index), that is representative of the push off ability, was significantly lower 
than CG mean value in both groups; after taking levodopa, APMax index improved only in PD patients.

\begin{tabular}{|l|l|l|l|l|l|}
\hline \multicolumn{2}{|l|}{ PD GROUP } & \multicolumn{2}{l|}{ PSP GROUP } & CG \\
\hline & $\begin{array}{l}\text { OFF } \\
\text { STATE }\end{array}$ & ON STATE & $\begin{array}{l}\text { OFF } \\
\text { STATE }\end{array}$ & ON STATE & \\
\hline Ankle Power & \multicolumn{7}{|l}{} & \\
\hline APMax (W/Kg) & $1.4(1.1)^{* *}$ & $3.2(0.8)^{*}$ & $1.1(0.7)^{* *}$ & $1.3(1.0)$ & $3.1(1.2)$ \\
\hline
\end{tabular}

Tab.3. Comparison of selected kinetic parameters (mean and standard deviation) for PD and PSP subjects (PD GROUP and PSP GROUP) and Control Group (CG) (*= $\mathrm{p}<0.05$, OFF versus ON states; $+=\mathrm{p}<0.05$ OFF state in PD GROUP versus OFF state in PSP GROUP; $* *=p<0.05$ compared with CG)

\subsection{Discussion}

The presented results of neurological (UPDRS) and functional (FIM) evaluations demonstrated significant differences in OFF and ON states of the two groups. Both PD and PSP patients revealed an improvement in terms of these two clinical scales. As concerns quantitative GA, our data highlighted that the treatment had significant effects only on PD group. The PD patients revealed improvements in most of spatio-temporal parameters (stance phase duration, anterior step length and velocity of progression), according to literature (Morris et al., 2001). As concerns kinematic parameters, significant changes were evidenced at all lower limb joints (ankle, knee and hip joints): they walked with ankle in a more normal biomechanical condition, especially at toe-off when it is able to reach values of plantarflexion close to normality, knee is characterised by a better flexion during swing phase and hip revealed an improved ability in extension during midstance. All the lower limb joints displayed a decrease of general stiffness and rigidity which is often present in PD after the treatment. In terms of ankle kinetic parameters, we observed that while the peak of ankle power generation (APMax index) improved in ON state, the same index normalised to the velocity of progression did not change significantly. This result reveals that the improvement of ankle propulsion capacity during terminal stance is directly connected to higher velocity of progression that patients are able to reach in ON state. On the contrary the same evaluation conducted on PSP patients revealed that no significant changes occurred after the levodopa therapy, in terms of spatio-temporal parameters, kinematic and kinetic indices. These results quantified the poor effect of this pharmacological response in these patients. As concerns the evaluation conducted using UPDRS and GA there is a contradiction in terms of obtained results, in particular for PSP patients. While in PD patients a significant improvement has been noted both in UPDRS score and in GA parameters, in PSP patients an improvement has been displayed in UPDRS score, but not in GA indices. These data confirmed that while UPDRS is recognised to be a valid method to evaluate PD patients, it is not satisfactory a satisfactory tool in PSP patients because it does not consider some features that are important in PSP and minor in PD (Golbe et al., 2007). 


\section{Conclusions}

The experience of the Posture and Motion Laboratory at the "San Raffaele Cassino" is described in the field of the use of GA in patients with PD and PSP. Our results demonstrated that quantitative GA may represent a precise, objective and reliable alternative to rating scales and commonly used tests in determining the dopaminergic response in patients with PD and PSP which provides a systematic evaluation to help in the early differentiation of PSP from other parkinsonian syndromes, like PD. Further studies should be conducted on this direction, with larger group of patients and considering sub-groups of patients, evaluating for example PD patients with walking problems or with freezing, in order to evaluate quantitatively if different responses to levodopa are found.

\section{References}

Davis, R. B.; Ounpuu, S.; Tyburski, D. J. \& Gage, J.R. A gait analysis data collection and reduction technique. Human Movement Science 1991; 10: 575-587

Fahn, S. \& Elton, R. Members of the UPDRS Development Committee. In: Fahn, S.; Marsden, C.D.; Calne, D.B. \& Goldstein M, eds. Recent Developments in Parkinson's Disease, Vol 2. Florham Park, NJ. Macmillan Health Care Information 1987, pp 15 3-163, 293-304

Gage, J.R. The treatment of gait problems in cerebral palsy. Gage JR editor. London: Cambridge University Press; 2004

Golbe, L.I. \& Ohman-Strickland, P. A clinical tasting scale for progressive supranuclear palsy. Brain 2007; 130: 1552-1565

Holmberg, B.; Johnels, B.; Ingvarsson, P., Eriksson, B. \& Resengren, L. CSFneurofilament and levodopa tests combined with discriminant analysis may contribute to the differential diagnosis of Parkinsonian syndromes. Parkinsonism and Related Disorders 2001; 8: 23-31

Keith RA, Granger CV, Hamilton BB, Sherwin FS. The Functional Independence Measure: a new tool for rehabilitation. In: Advances in clinical rehabilitationEisenberg MG, Grzesiak RC, eds. (1987) New York: Springer. 6-18

Lubik, S.; Fogel, W.; Tronnier, V.; Krause, M.; Konig, J. \& Jost, W.H. Gait analysis in patients with advanced Parkinson disease: different or additive effects on gait induced by levodopa and chronic STN stimulation. Journal of Neural Transmission 2006; 113: 163-173

Moore, S.T.; MacDougall, H.G.; Gracies, J.M.; Cohen, H.S. \& Ondo, W.G. Locomotor response to levodopa in fluctuating Parkinson's disease. Exp Brain Res 2008; 184: 469-478

Morris, M.E.; Huxham, F.; McGinley, J.; Dodd, K. \& Iansek, R. The biomechanics and motor control of gait in Parkinson disease. Clinical Biomechanics 2001; 16: 459-470

Vokaer, M.; Abou Azar, N. \& Zegers de Bayl, D. Effects of levodopa on upper limb mobility and gait in Parkinson's disease. Journal of Neurology, Neurosurgery and Psychiatry 2003; 74: 1304-1307 